\title{
Long-term photometric study of FK Comae Berenices and HD 199178^
}

\author{
K. Panov and D. Dimitrov \\ Institute of Astronomy, Bulgarian Academy of Sciences, Tsarigradsko shossee 72, 1784 Sofia, Bulgaria \\ e-mail: [kpanov;dinko]@astro.bas.bg \\ Received 12 May 2006 / Accepted 25 January 2007

\section{ABSTRACT}

\begin{abstract}
Aims. The rapidly rotating active stars FK Com and HD 199178 are investigated using extensive long-term photometry. The data set includes previously unpublished photometry, obtained at the Rozhen National Astronomical Observatory. We look for periodicities in the long-term photometric behaviour of both stars, as well as for spots, using light curve modelling. Special attention is paid to the behaviour of the "flip-flop" phenomenon.

Methods. Light curves in UBV of FK Com and HD 199178 are used to construct an O-C diagram for each star, and they are modelled to obtain spot distribution.

Results. The main result for FK Com is that the "flip-flop" phenomenon is a superposition of an oscillating process of activity and real and fast "flip-flops". For the oscillating component, we obtained a cycle of 5.8 years. The same cycle is also present in other photometric characteristics.

The O-C diagram for HD 199178 shows a cycle of 4.2 years. Modelling of the light curves of both stars reveals a large high-latitude spot on each star and also smaller spots in the equatorial belt or at intermediate latitudes.
\end{abstract}

Key words. stars: activity - stars: individual: FK Comae Berenices - stars: individual: HD 199178 - stars: starspots

\section{Introduction}

FK Com and HD 199178 belong to a small group of G-K stars with remarkable activity and extremely rapid rotation (Bopp \& Rucinski 1981). Their evolutionary status is not yet clear. FK Com is a G2III to G7III star, apparently a single giant with $v \sin i=162.5 \mathrm{~km} \mathrm{~s}^{-1}$ (Huenemoerder et al. 1993). Its chromospheric activity is similar to the chromospheric activity of the RS CVn stars. Strong emission has been observed in $\mathrm{H} \alpha$ and $\mathrm{Ca}$ II H+K lines (Bopp \& Stencel 1981; Bopp 1982). Variability in the $\mathrm{H} \alpha$ emission has been reported by Welty \& Bopp (1993). Chromospheric flares have been observed in $\mathrm{H} \alpha$ (Dorren et al. 1984; Olivera \& Foing 1999) and in broad band filters. Photometric variability, attributed to rotational modulation by photospheric spots was first reported by Chugainov (1966). He also determined the photometric period, $P=2$ d.412, which is the period of rotation. Welty \& Ramsey (1994) detected the X-ray emission of FK Com and radio observations were reported by Rucinski (1991) and by Welty \& Ramsey (1994). It is a common belief that the strong activity of FK Com is due to its rapid rotation. Considering the evolutionary status of the star, the rapid rotation of FK Com is difficult to understand. Walter \& Basri (1982) suggested that the star may be accreting material from a small and unseen companion. However, attempts to reveal the unseen companion were unsuccessful (Huenemoerder et al. 1993).

Another possible scenario is a coalesced binary system (Webbink 1976). Jetsu et al. (1991, 1993, 1994b) revealed

* Tables 2, 3, and 5 are only available in electronic form at the CDS via anonymous ftp to cdsarc.u-strasbg.fr (130.79.128.5) or via

http://cdsweb.u-strasbg.fr/cgi-bin/qcat? J/A+A/467/229 a new and interesting phenomenon: a "flip-flop" occurs in the FK Com activity, and it is related to two active longitudes, apparently situated at opposite longitude sites on the photosphere. The next question to be solved was: what is a flip-flop? Is it a switch over of activity from one active longitude to the opposite one, or is there a spot migration on the surface? Doppler imaging but also a long-term set of light curves could be used to look for the answer. Korhonen et al. (1999, 2000, 2001b, 2002) used the Doppler imaging technique to conclude that the flipflop is a rapid change in activity from one active longitude to the opposite one and not a movement of spots. This would also imply that minimum light would always occur around phases 0.0 and 0.5 , assuming that the two active longitudes are permanent.

This conclusion seemed at first to be fulfilled (see e.g. Fig. 4 in Jetsu et al. 1993). With the data presently available, however, this conclusion is no longer valid and there is evidence of spot migration.

The star HD 199178 (=V1794 Cygni) is another rapidly rotating giant of the FK Comae type stars (Bopp \& Stencel 1981). The main characteristics for such a classification are: spectral class G5III-IV (Herbig 1958) and $v \sin i=80 \mathrm{~km} \mathrm{~s}^{-1}$ (Herbig 1958; Huenemoerder 1986). It is also apparently single with an upper limit to the radial velocity variation $\pm 2 \mathrm{~km} \mathrm{~s}^{-1}$ (Huenemoerder 1986). The photometric period 3.337 was determined by Bopp et al. (1983), which is the period of rotation. Jetsu et al. (1990) improved the period to 3.337484, claiming that phase consistence has been achieved for 13 years. In the same study, Jetsu et al. (1990) determined long-term cycles: $9.07 \mathrm{yr}$ for the mean brightness and $2.84 \mathrm{yr}$ for the changes in amplitude of rotational modulation. In a later investigation, however, Jetsu et al. (1999a) denied the significance of both the activity cycles found previously. They also denied the earlier constant 
Table 1. Rozhen observing log of FK Com and HD 199178.

\begin{tabular}{|c|c|c|c|c|c|c|c|c|c|c|c|c|c|c|}
\hline Time range & $n$ & $N_{V}$ & $N_{B}$ & $N_{U}$ & Time range & $n$ & $N_{V}$ & $N_{B}$ & $N_{U}$ & Time range & $n$ & $N_{V}$ & $N_{B}$ & $N_{U}$ \\
\hline \multicolumn{15}{|c|}{ FK Com } \\
\hline 12.03.-20.04.91 & 4 & 5 & 5 & 5 & 13.03.-09.05.00 & 5 & 35 & 35 & 34 & 13.07.-28.07.03 & 8 & 20 & 19 & 18 \\
\hline 19.05.-30.06.91 & 8 & 12 & 11 & 10 & $04.06 .-04.07 .00$ & 10 & 16 & 16 & 16 & 19.11.-21.12.03 & 9 & 19 & 19 & 19 \\
\hline 08.07.-19.07.91 & 4 & 5 & 4 & 3 & 15.03.-19.04.01 & 4 & 11 & 11 & 10 & 31.01.-18.02.04 & 7 & 30 & 30 & 30 \\
\hline 05.08.-12.09.91 & 4 & 5 & 4 & 2 & 03.06.-11.06.01 & 6 & 25 & 23 & 21 & 22.04 .04 & 1 & 6 & 6 & 6 \\
\hline 09.02.-04.04.92 & 6 & 22 & 23 & 22 & 29.06.-09.07.01 & 5 & 12 & 10 & 8 & 15.06.-18.07.05 & 6 & 14 & 14 & 14 \\
\hline $10.05 .-11.05 .92$ & 2 & 15 & 15 & 15 & 09.02.-26.04.02 & 4 & 15 & 15 & 14 & 17.11.-07.01.05 & 5 & 8 & 8 & 8 \\
\hline 07.06.-30.08.92 & 6 & 7 & 5 & 2 & 22.05.-24.07.02 & 7 & 15 & 15 & 13 & 06.02.-27.04.05 & 10 & 37 & 37 & 36 \\
\hline 19.02.-25.04.93 & 9 & 25 & 25 & 25 & 26.12.-14.03.03 & 10 & 88 & 86 & 82 & 29.06.-10.08.05 & 7 & 14 & 12 & 12 \\
\hline 24.06.-24.08.93 & 5 & 5 & 4 & 2 & 28.04.-01.05.03 & 3 & 33 & 33 & 30 & & & & & \\
\hline 26.05.-24.08.95 & 6 & 6 & 5 & 3 & 27.05.-02.07.03 & 13 & 41 & 41 & 37 & Total & 174 & 546 & 531 & 497 \\
\hline \multicolumn{15}{|c|}{ HD 199178} \\
\hline 09.07.-10.07.91 & 2 & 2 & 2 & 2 & 01.10.-03.10.96 & 3 & 2 & 3 & 3 & 11.07.-28.07.03 & 10 & 22 & 22 & 22 \\
\hline 10.09.-09.10.91 & 8 & 8 & 8 & 8 & 22.09.-10.10.97 & 4 & 4 & 4 & 4 & 15.08.-31.08.03 & 10 & 41 & 41 & 39 \\
\hline 25.08.-30.08.92 & 6 & 13 & 13 & 13 & 20.08 .98 & 1 & 1 & 1 & 1 & 20.09.-28.09.03 & 7 & 23 & 23 & 22 \\
\hline 20.08.-04.09.93 & 8 & 9 & 9 & 9 & 01.08.-05.08.01 & 4 & 6 & 6 & 6 & 20.11.-20.12.03 & 7 & 18 & 17 & 16 \\
\hline 19.08.-31.08.94 & 8 & 14 & 14 & 14 & 27.08.-23.09.01 & 7 & 13 & 13 & 13 & 19.06.04 & 1 & 1 & 1 & 1 \\
\hline 17.08.-23.08.95 & 3 & 3 & 3 & 3 & 23.05 .02 & 1 & 1 & 1 & 1 & 21.08.-08.09.04 & 7 & 23 & 22 & 22 \\
\hline 15.09.-13.10.95 & 8 & 9 & 9 & 9 & 01.06.-24.07.02 & 4 & 6 & 6 & 6 & 18.11.-24.11.04 & 4 & 9 & 9 & 8 \\
\hline 17.08.-25.08.96 & 5 & 5 & 5 & 5 & 16.10.-01.11.02 & 3 & 9 & 9 & 9 & Total & 121 & 241 & 240 & 235 \\
\hline
\end{tabular}

Note: $n$ - number of nights; $N_{V}, N_{B}, N_{U}$ - number of data points.

period ephemeris approach, stating that light curves in different observing seasons could be modelled only with different (though similar) periods. This in fact means a random shift in longitude of the active (spot) region on the stellar surface. Other values for the photometric period have been suggested, making the situation even more complicated. Flip-flop has been found in a number of other stars (Berdyugina \& Tuominen 1998; Berdyugina et al. 1999). For a summary, see Table 1 in Elstner \& Korhonen (2005). From the rotational modulation of polarization, Huovelin et al. (1987) determined an inclination (of rotational axis) of $i=79^{\circ}$.

From this introduction, it is clear that many questions remain concerning the long-term photometric behaviour of both FK Com and HD 199178, and this we address in the present study.

\section{Observations}

This study includes previously unpublished data for FK Com and HD 199178, obtained with the $60 \mathrm{~cm}$ telescope of the Rozhen National Astronomical Observatory and the singlechannel, photon-counting photometer. The Rozhen observing $\log$ is shown in Table 1. For FK Com, standard $U B V$ differences are obtained with respect to the comparison star HD 117567. The check star is HD 117876. For HD 199178, the comparison star is HD 199206 and the check HD 199312. Data for the comparison and check stars can be found in Jetsu et al. (1994a, 1999a). Four consecutive 10s integrations have been averaged to obtain a single data point, and a mean of 3-5 individual data has been calculated. Care was taken in the reduction procedure to account for dead-time correction, extinction and the instrumental system corrections.

The Rozhen data for FK Com is added to the published photometry (Jetsu et al. 1994a,b; Heckert 1993; Strassmeier et al. 1997; Korhonen et al. 2001a, 2002) to construct a data set in $V$ covering 1966-2005 (Table 2a). For HD 199178, a data set was constructed, using published sources (Jetsu et al. 1999a; Strassmeier et al. 1999) and Rozhen photometry, covering 1977-2004 (Table 2b). Tables 2a,b contain: Col. 1 - subset number, Cols. 2 and 3 - Julian Date for beginning and end of observing run, and Col. 4 - reference to published photometry. Tables 3a,b contain the Rozhen $U B V$ photometry for FK Com and HD 199178, respectively: Col. 1 - Julian Date, Cols. 2-4 $V, B$ and $U$ magnitudes in the Johnson system and the last three columns contain the respective errors in $V, B$, and $U$. (Tables 2 and 3 are only available in electronic form at the CDS.)

\section{Light curve modelling}

In order to look for the distribution of spots, we modelled the light curves for the whole data set, using the technique of the synthetic light curves, described by Eker (1994), and a computer code, written by D. Dimitrov. In Table 4, input parameters of the modelling are listed, taken from published sources. The temperature difference is $\Delta T=T_{\text {phot }}-T_{\text {spot }}$. Inclination $i$ of rotational axis is taken from Korhonen et al. (1999, 2000) and Strassmeier et al. (1999). The unspotted magnitude is $V_{\max }$. Limb-darkening coefficients are taken from Al-Naimiy (1978) and a linear limb-darkening law was applied, assuming the same law for the unspotted photosphere and for the spots.

The standard deviation of the residuals (see Tables 5a,b, only available in electronic form at the CDS) we reckoned from

$\sigma=\sqrt{\frac{\sum_{i=1}^{n}\left(\mathrm{O}_{i}-\mathrm{C}_{i}\right)^{2}}{(n-1)}}$

where $\left(\mathrm{O}_{i}-\mathrm{C}_{i}\right)$ are the residuals between observed and synthetic light curves.

The free parameters for constructing a light curve are: the number of spots, coordinates, and sizes of each spot. For a set of parameters, the solution for the light curve was obtained and the standard deviation $\sigma$ computed. Then a number of iterations were done, changing parameters one by one, step by step, until the desired fit is obtained with the minimal number of spots. Spots of sizes less than $5^{\circ}$ or spots of latitude $\leq-30^{\circ}$, were discarded. The best solutions obtained are listed in Tables 5a,b for FK Com and HD 199178, respectively. Columns in Table 5 include: data subset, year, parameters of the synthetic 
Table 4. Adopted parameters for light curve modelling.

\begin{tabular}{llll}
\hline \hline \multicolumn{2}{c}{ Parameter } & FK Com & HD 199178 \\
\hline$T_{\text {phot }}$ & {$[\mathrm{K}]$} & 5080 & 5450 \\
$\Delta T$ & {$[\mathrm{~K}]$} & 1200 & 1500 \\
$i$ & $\left.{ }^{\circ}\right]$ & 60 & 40 \\
$V_{\max }$ & {$[\mathrm{mag}]$} & 8.03 & 7.045 \\
$u$ & & 0.71 & 0.67 \\
$\mathrm{HJD}_{0}$ & [days] & 2439252.895 & 2442001.5 \\
$P$ & {$[$ days $]$} & 2.4002466 & 3.31925 \\
\hline
\end{tabular}

light curve: $V_{\min }, V_{\max }, V_{\text {mean }}$, amplitude in $V, \mathrm{JD}$, and phase of minimum light, spot longitude, spot latitude, and spot size. The last column of Table 5 gives $\sigma$. For FK Com, 162 light curves from the total of 177 subsets (Table 2a) were constructed. The analysis include the light curves in 1979-2005, because data is scarce prior to 1979. For HD 199178, 148 light curves from the total of 169 subsets (Table 2 b) were constructed and analysed for 1977-2004. Individual light curves were determined in such a way that time resolution was achieved with reasonable phase coverage.

\section{Data analysis and results}

\subsection{FK Com}

We first address the question of minimum light behaviour. In Fig. 1, the $\mathrm{O}-\mathrm{C}$ diagram is shown using the ephemeris of Jetsu et al. (1993):

$$
\mathrm{HJD}_{\phi_{\text {min }}}=2439252.895+2.4002466 E \text {. }
$$

The flip-flop phenomenom has been detected photometrically by sudden jumps in phase of the light-curve minimum (Jetsu et al. $1991,1993,1994 b)$ by $180^{\circ}$, which led to assuming two active longitudes being activated alternatively. However, with more data accumulated, the picture became more complicated, and there is increasing evidence that the light curve minimum is moving in phase. From Fig. 1, it is apparent that the phase of minimum light (in $V$ ) is not always in position 0.0 or 0.5 , as required by the simple flip-flop hypothesis. On the contrary, the variation in phases seems to be an oscillatory process, occasionally interrupted by sudden jumps (flip-flop) in phase. Flip-flop events are clearly seen in 1985.3 (No. 1), 1988.7 (No. 2), 1997.7 (No. 3), 1998.25 (No. 4), 1998.95 (No. 5), and 1999.3 (No. 6). The flip-flops Nos. 1 and 2 have been reported by Jetsu et al. (1993, 1994b) and Korhonen et al. (2002).

To look for the spots that cause the flip-flop events, modelling of the light curves of FK Com was carried out (see Sect. 3). For FK Com, the best solution is achieved with 3 spots. In Table 5a, longitude, latitude and size of the detected spots are given. The largest Spot 1 is present in all 162 subsets (latitude generally higher than $70^{\circ}$, size $\sim 30-45^{\circ}$ ). Spot 2 is detected in 149 subsets, and Spot 3 only in 39 subsets. Spots 2 and 3 are much smaller than Spot 1 (each size is $\sim 5-25^{\circ}$ ). In Fig. 2, we plotted the longitude of Spot 1 (upper panel), and Spots 2 and 3 in the lower panel, respectively. The same position of each spot is plotted three times in the ordinate, in order to allow for better understanding of the variations observed. From Fig. 2, it is apparent that only the longitudes of Spot 1 show oscillatory behaviour, obviously corresponding to the $\mathrm{O}-\mathrm{C}$ diagram in Fig. 1.

The close correlation of the $\mathrm{O}-\mathrm{C}$ diagram with the longitude variation of Spot 1 is strong evidence of the migration of Spot 1 . The oscillations proceed between two extreme positions

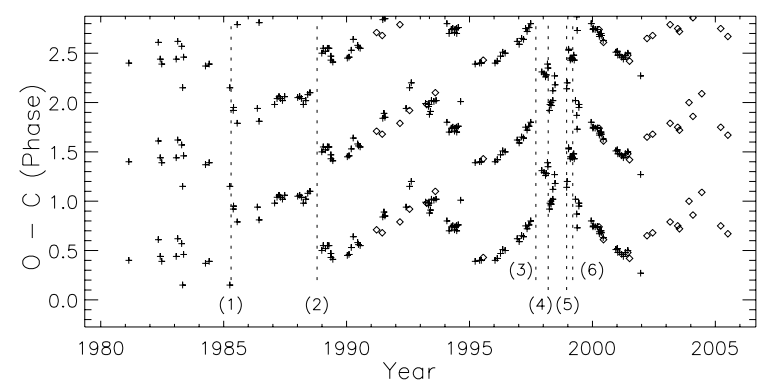

Fig. 1. Phases of photometric minima of FK Com. Diamonds denote Rozhen data and crosses show published data. Phases are reckoned from Eq. (2). Vertical lines denote flip-flop events.

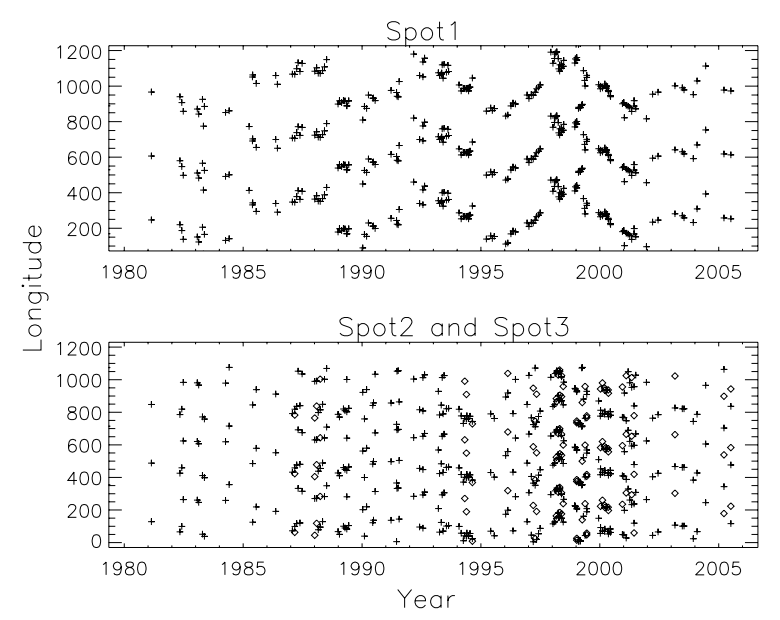

Fig. 2. Longitudes of Spot 1, Spot 2 (crosses), and Spot 3 (diamonds) of FK Com.

in longitude, which are separated by about $180^{\circ}$. The flip-flop events Nos. 1-2 (further on we take designation f-f) correspond to a sudden switch over from one extreme position in longitude to the other one. The oscillatory manner of the Spot 1 longitude variation points to the existence of a whole "path of activity" and to a migrating Spot 1 along this path. Fourier analysis, using the PERIOD04 program (Lenz \& Breger 2005), reveals a $5.8 \pm$ $0.1 \mathrm{yr}$ cycle for the oscillating activity process. Looking for periodicities in the flip-flop events, Korhonen et al. (2001a) found a possible cycle of 6.4 years, together with other possible cycles. The interpretation of the 5.8 years cycle is given here in the context of an oscillatory activity process, valid during the 25 years of observations. The same $5.8 \mathrm{yr}$ cycle is present in the variation in the Spot 1 longitudes (see Fig. 6). This is a clear indication that Spot 1 is the dominant factor of the FK Com photometric behaviour. For f-f 3, 4, 5, and 6 (Fig. 1), the picture seems more complicated, with contributions from Spot 1 (in each case), but also from Spots 2 or 3 . The jumps in phase in these cases are different from $180^{\circ}$. The longitude variation does not necessarily imply that the spot migration proceeds only in the longitude direction. Latitudinal migration of Spot 1 and differential rotation could not be ruled out, as it would also result in a longitude variation. Evidence of differential rotation was found by Korhonen et al. (2002). However, because of insufficient latitudinal resolution of the synthetic light-curve method, it is not possible at present to distinguish between the two possibilities for Spot 1 movement: longitudinal migration or latitudinal migration with differential rotation.

Figure 3 shows the temporary behaviour of minimum light, maximum light, mean light, and the amplitude of rotational 


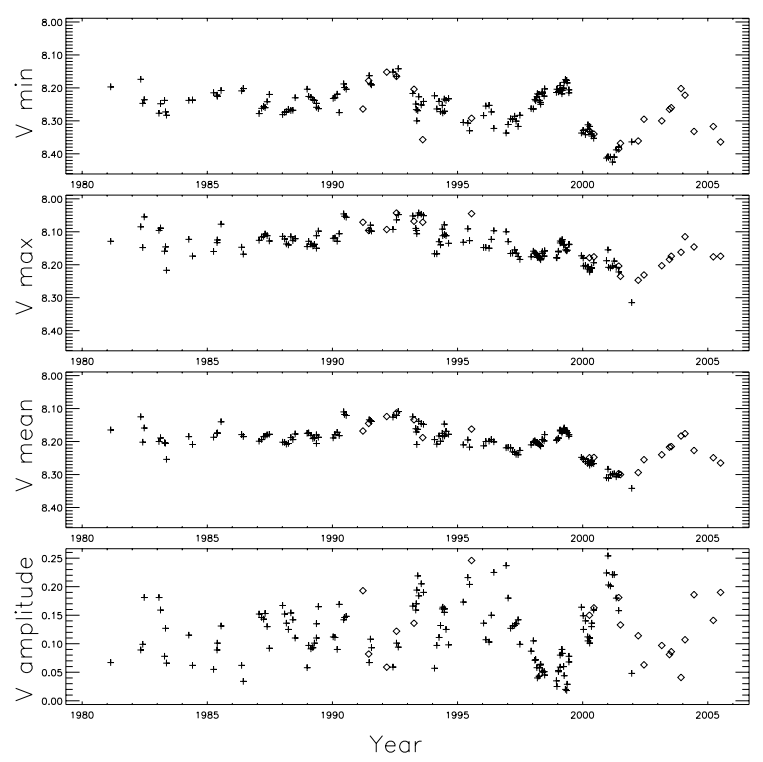

Fig. 3. Minimum, maximum, mean light, and amplitude of rotational modulation of FK Com. Symbols as in Fig. 1.

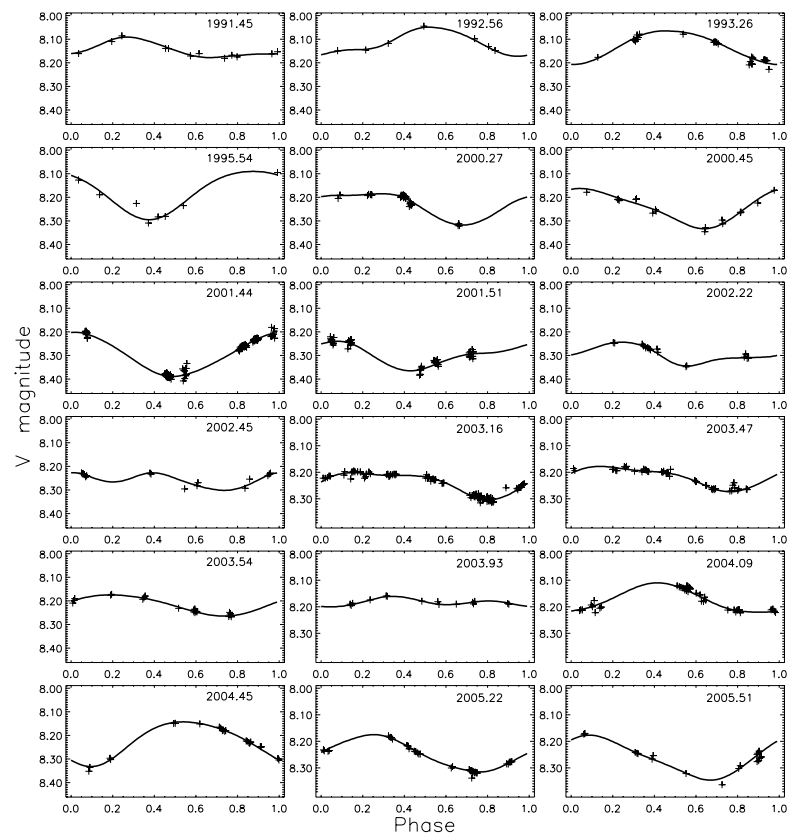

Fig. 4. Rozhen photometry of FK Com.

modulation. In Fig. 4, light curves of FK Com with Rozhen data are shown, in filter $V$. Figure 5 represents the long-term photometric behaviour of FK Com with the whole data set. A period search with the PERIOD04 program was carried out with the long-term variation of minimum light, maximum light, mean light, the amplitude of rotational modulation, the $\mathrm{O}-\mathrm{C}$ diagram, and the longitude variation of Spots 1 and 2. All power spectra are shown in Fig. 6. The first column in Fig. 6 contains the spectra for 1966-1990, the second column is for 1990-2005, and the last column is for the whole observation period. Clearly seen are the peaks at 5.8 years in the $\mathrm{O}-\mathrm{C}$ and in the longitude of Spot 1. The presence of the same cycle in the minimum and the mean light may also be seen in the second column, i.e. for 1990-2005. The case with the amplitude of rotational modulation is not very clear, because of the broad peaks in Cols. 2 and 3. However, this could possibly be an indicator of the presence of

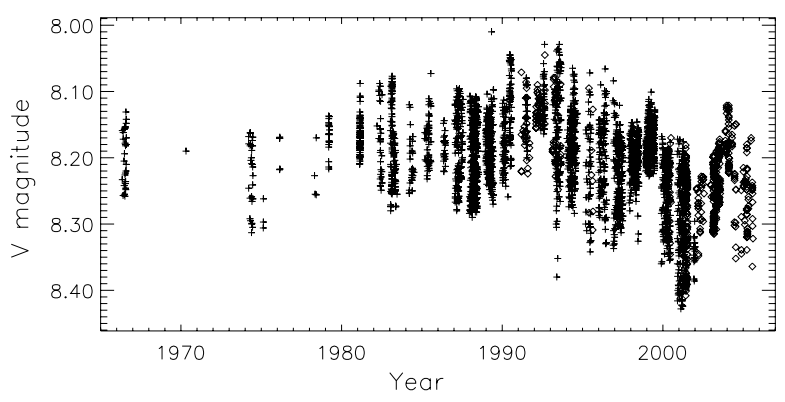

Fig. 5. Photometry of FK Com in $V$. The vertical spread of data is due to rotational modulation. Symbols as in Fig. 1.

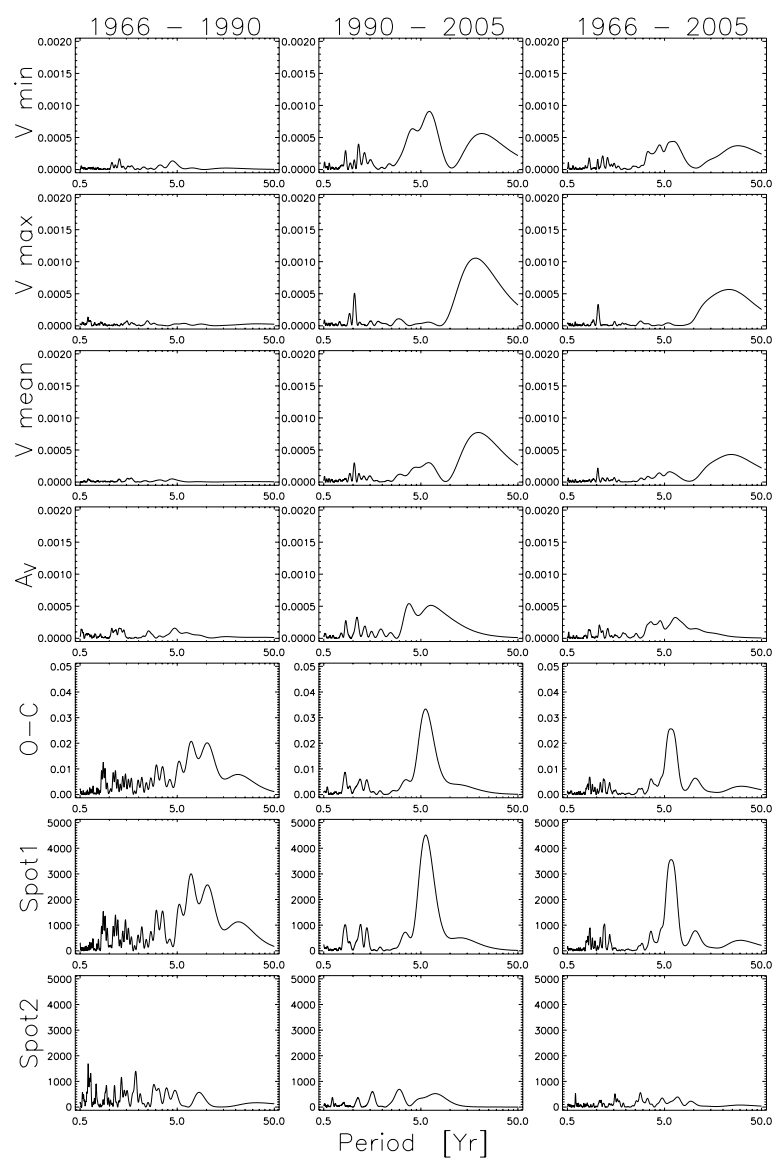

Fig. 6. Power spectrum of FK Com with: minimum light, maximum light, mean light, amplitude of rotational modulation, O-C diagram, longitude of Spot 1, and longitude of Spot 2.

the same cycle, too. From Fig. 6, it could be concluded that the 5.8 years cycle is dominating the whole photometric behaviour of FK Com in at least 1990-2005. The $5.8 \mathrm{yr}$ cyclic variation of the FK Com $\mathrm{O}-\mathrm{C}$ diagram follows the ephemeris (in days):

$\mathrm{JD}\left(\mathrm{O}-\mathrm{C}_{\min }\right)=2439420+2118 E \quad$ [days].

To explain the flip-flop phenomenon, Elstner \& Korhonen (2005) assume a non-axisymmetric dynamo mode, together with an oscillating axisymmetric field. The non-axisymmetric modes are assumed to produce two active longitudes $180^{\circ}$ apart, and the axisymmetric modes are responsible for the oscillations.

The situation presented here (Fig. 1) is different and favours the spot migration interpretation. Due to some oscillatory activity, Spot 1 seems to be oscillating in longitude but, occasionally, there are interruptions (flip-flops) in the oscillatory process. 
Table 6. Flares of FK Com.

\begin{tabular}{lccccc}
\hline \hline Flare & $\mathrm{HJD}_{\mathrm{fl}}$ & $\Delta \phi$ & $(B-V)_{\mathrm{fl}}$ & $(U-B)_{\mathrm{fl}}$ & $A_{U}(\mathrm{fl})$ \\
\hline 1 & 2448679.521 & 0.32 & -0.024 & -0.054 & 0.081 \\
2 & 2452391.491 & 0.29 & -0.036 & -0.468 & 0.507 \\
3 & 2452713.506 & 0.20 & -0.023 & -0.079 & 0.096 \\
\hline
\end{tabular}

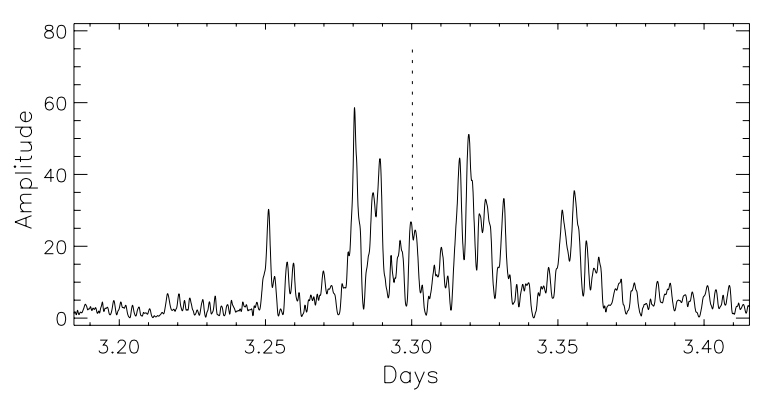

Fig. 7. Power spectrum of HD 199178. The vertical line denotes the photometric period, $P=3.30025$.

We should also address the question of the stability of spots. Obviously, Spot 1 is not destroyed by differential rotation, and it has survived during the whole set of observations, i.e. at least for 1979-2005. Spot 2 is seen sporadically in the analysis in 1979-2005. Spot 3 emerged after 1987 and is seen sporadically until 2005.

During the Rozhen observations, 3 flares were recorded, which are presented in Table 6 . The phase difference in Table 6 is $\Delta \phi=\phi_{\text {flare }}-\phi_{\text {Spot } 1}$, and it indicates the relative position of the flare (only in longitude) with respect to the largest Spot 1. It should be noted, however, that no information could be obtained for the flare latitude.

\section{2. $H D 199178$}

The photometric study of HD 199178 (V1794 Cyg) proceeded a similar way as for FK Com. For the whole data set, 1977-2004, phases of minimum light were calculated, using the ephemeris:

$\mathrm{HJD}_{\phi_{\min }}=2442002.8+3.30025 \mathrm{E}$.

The period chosen is a "mean" one in the sense that a systematic drift of phases is avoided. In Fig. 7, we plotted the power spectrum of HD 199178 around the chosen period. The complexity of the diagram is obvious and due to both the data accuracy and the very real variations in the photometric period. This answers the question of why previous investigators published many different values for the photometric period of HD 199178 (Jetsu et al. 1990, 1999b; Strassmeier et al. 1999; Hackman et al. 2001). In a case with a variable period, such as HD 199178, the highest peak in the power spectrum diagram is not necessarily the best choice for the period. The variability of the photometric period is seen in Fig. 8, where the $\mathrm{O}-\mathrm{C}$ diagram is shown. From Fig. 8, it is apparent that the $\mathrm{O}-\mathrm{C}$ reveals a periodic (cyclic) behaviour. In order to look for the spots on HD 199178, all light curves (data from Table 2b) were modelled, and the results (spot longitude, latitude, and size) are listed in Table 5b. The modelling reveals a large (size $15^{\circ}-30^{\circ}$ ) Spot 1 , lasting at least during 1980-2004 (Spot 1 is seen in 148 light curves). The best solution also includes two smaller spots $\left(\right.$ size $\left.5^{\circ}-20^{\circ}\right)$ : Spot 2 is detected in 120 light curves and Spot 3 is seen in only 19 light curves. Spot 1 is always a high-latitude (polar) spot.

In Fig. 9, the longitudes of the three detected spots are shown. Clearly seen are the variations in the longitude of Spot 1 ,

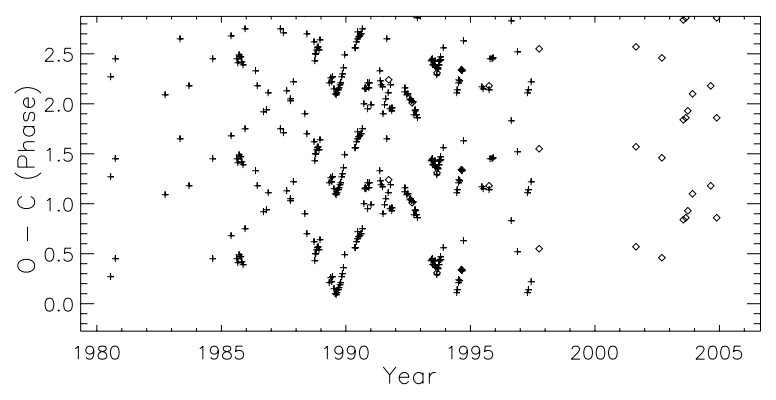

Fig. 8. Phases of the photometric minima of HD 199178. Diamonds denote Rozhen data and crosses are from published data. Phases are reckoned from Eq. (4).

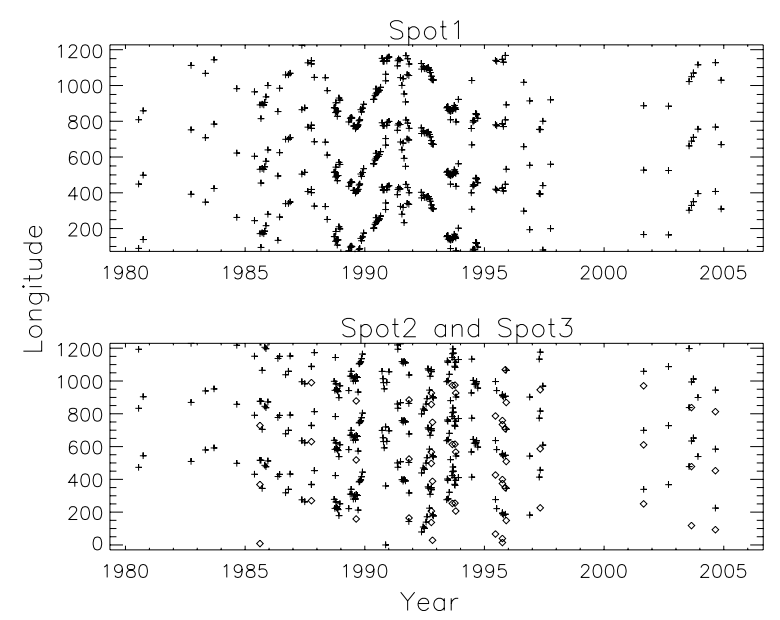

Fig. 9. Longitudes of Spot 1, Spot 2 (crosses), and Spot 3 (diamonds) of HD 199178.

closely corresponding to the variations in the $\mathrm{O}-\mathrm{C}$ diagram. The extreme positions in longitude of Spot 1 are about $400^{\circ}$ apart. The situation looks somewhat similar to FK Com, but the amplitude of the $\mathrm{O}-\mathrm{C}$ variation is larger and there is no evidence of a flip-flop in HD 199178.

A period search with the PERIOD04 program was carried out, and we analysed the $\mathrm{O}-\mathrm{C}$ variations and the longitude variations of Spots 1 and 2. The period search reveals a cycle of $4.2 \pm$ 0.2 years for the $\mathrm{O}-\mathrm{C}$ and the same cycle for the longitude variations of Spot 1 (Fig. 13). The result for the longitude of Spot 2 is inconclusive - a broad peak in the power spectrum. Our period search with other photometric characteristics (minimum light, maximum light, mean light and amplitude of the rotational modulation) was unsuccessful: we found no periodicity. Jetsu et al. (1990) reported a $9.07 \mathrm{yr}$ cycle in mean light and a $2.8 \mathrm{yr}$ cycle in the amplitude of the photometric variations. Neither of these cycles could be confirmed by the present study. Within the errors, both the $\mathrm{O}-\mathrm{C}$ variations and the variations of the longitude of Spot 1 have the same cycle: 4.2 years. The variations observed could be explained by Spot 1 migration, either in longitude or in latitude. In the second case, differential rotation is assumed. The 4.2 yr. cyclic variation in the O-C diagram of HD 199178 follows the ephemeris (in days):

$\mathrm{JD}\left(\mathrm{O}-\mathrm{C}_{\min }\right)=2441460+1535 E \quad$ [days].

Figure 10 shows the time-variations of the $V_{\min }, V_{\max }, V_{\text {mean }}$, as well as the amplitude of rotational modulation. In Fig. 11, some light curves in $V$ from Rozhen are shown. The long-term photometric behaviour is plotted in Fig. 12, for the whole period of observation. 


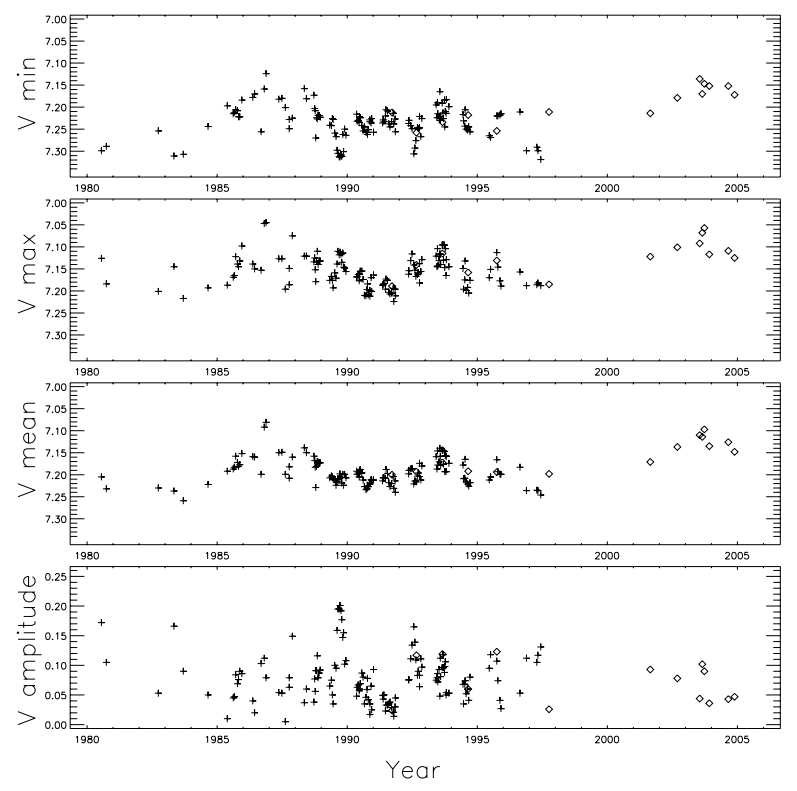

Fig. 10. Minimum, maximum, mean light, and amplitude of rotational modulation of HD 199178. Symbols as in Fig. 8.

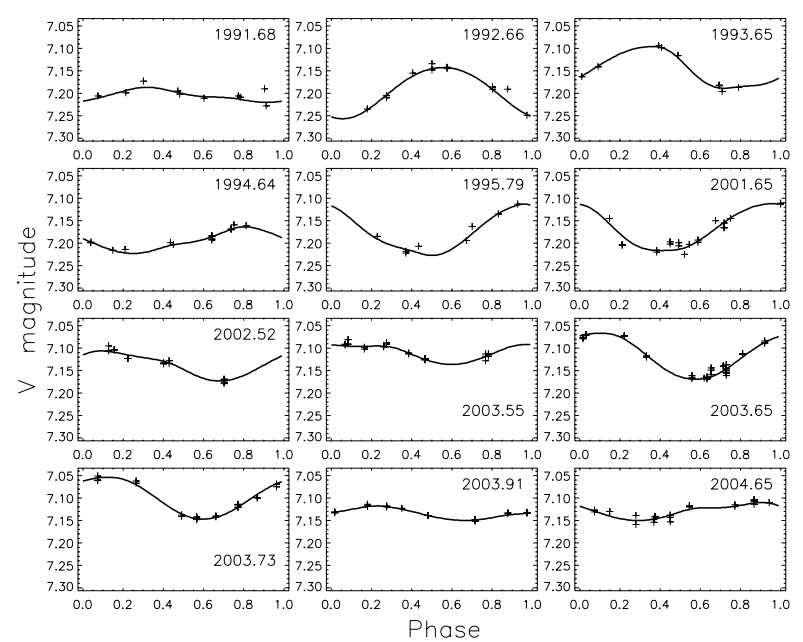

Fig. 11. Rozhen photometry of HD 199178.

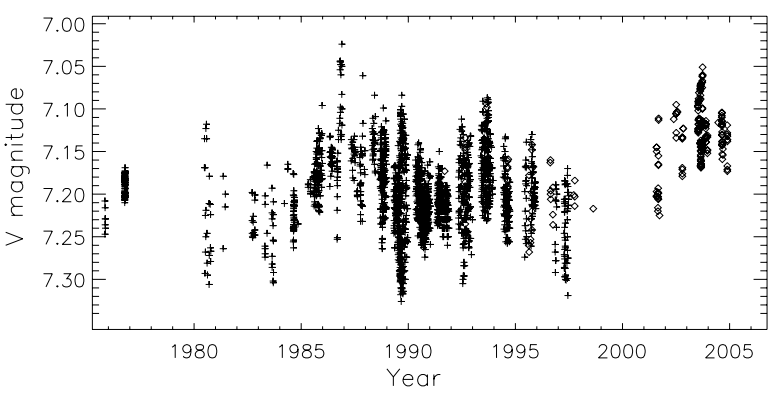

Fig. 12. Photometry of HD 199178 in $V$. The vertical spread of data is due to rotational modulation. Symbols as in Fig. 8.

\section{Uniqueness of solution and accuracy of the synthetic light curves}

Uniqueness is most important when discussing spot models. The problem of the uniqueness of the synthetic light curves solution has been discussed by Eker (1994, 1995, 1996, 1999a,b). Generally, an ambiguity arises from the assumed "spotless

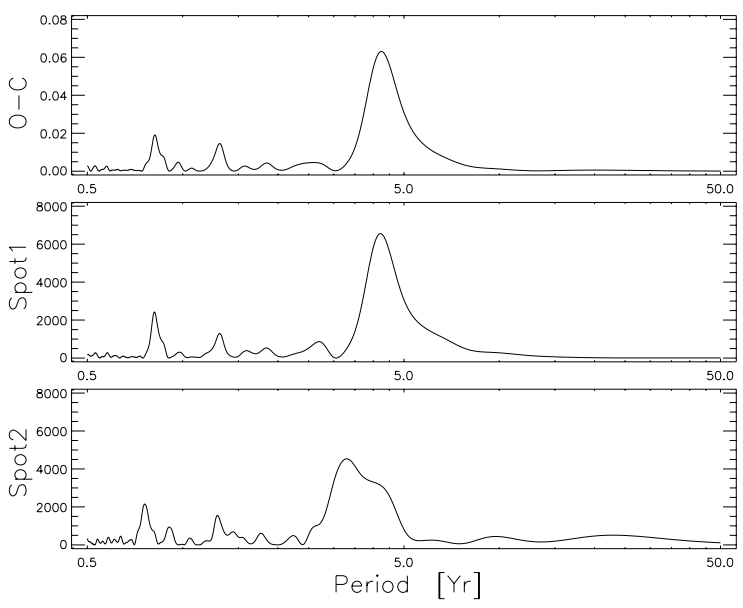

Fig. 13. Power spectrum of HD 199178 with: O-C diagram, longitude of Spot 1 and longitude of Spot 2.
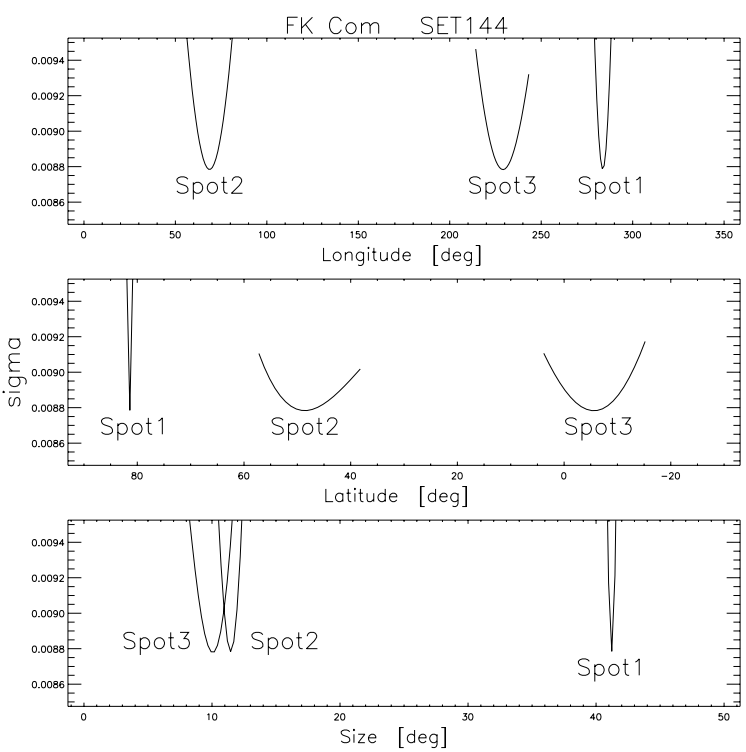

Fig. 14. Accuracy of the FK Com light curve modelling, using SET144. The function $\sigma$ is shown versus spot longitude (upper panel), spot latitude (middle), and spot size (lower panel).

magnitude". Even with an observing data set, lasting for several decades, one can never be sure about the "spotless magnitude". Another ambiguity arises from the fact that light curves cannot distinguish between a large polar spot and a number of smaller spots in the equatorial belt (Poe \& Eaton 1985). In the case of FK Com, there is evidence of a large polar spot, obtained by Doppler imaging (Korhonen et al. 1999, 2000).

According to the extensive study of Eker (1999a), synthetic light curves are a unique function of the parameters. Thus, if there is a way to distinguish between a polar spot and a belt, the synthetic light curve solution is unique.

The accuracy of the solution depends of course on the accuracy of the observing photometry. This, however, should not be mixed up with non-uniqueness. In our models, we have fixed the inclination $i$, the temperature of the photosphere, and the spots, for each star (Table 4). We carried out an analysis of the accuracy of solution for the spot's longitude, latitude, and size, using for FK Com light curve of SET144 and for HD 199178 light curve of SET145. The results are shown in Fig. 14 for FK Com, in Fig. 15 for HD 199178, and in Table 7. In Figs. 14 and 15, the 

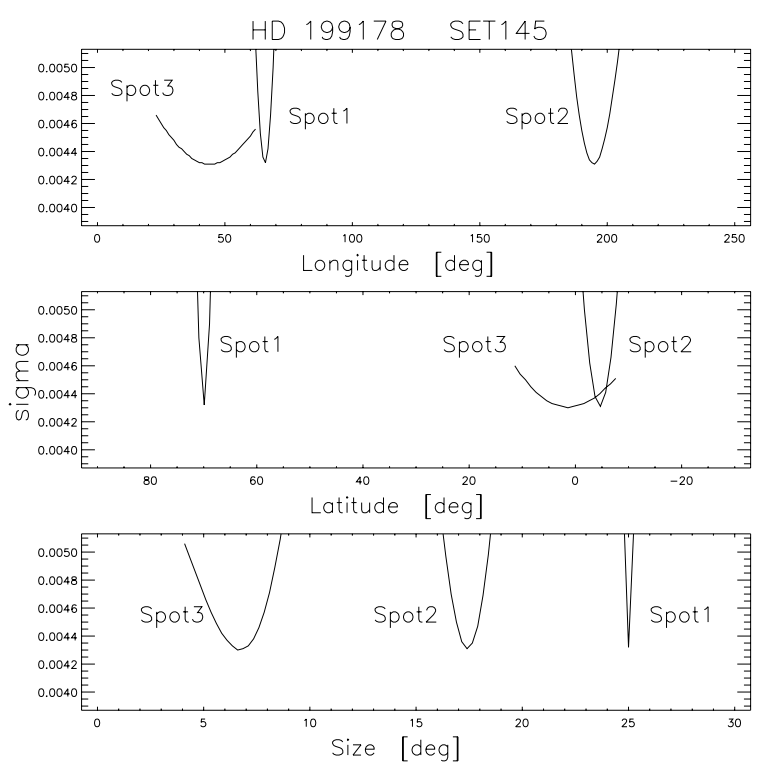

Fig. 15. Accuracy of the HD 199178 light curve modelling, using SET145. Panels are as in Fig. 14.

Table 7. Accuracy of the light curve solutions.

\begin{tabular}{cccc}
\hline \hline$\Delta \sigma=0.0001$ & $\begin{array}{c}\Delta l \\
{[\mathrm{deg}]}\end{array}$ & $\begin{array}{c}\Delta b \\
{[\mathrm{deg}]}\end{array}$ & $\begin{array}{c}\Delta r \\
{[\mathrm{deg}]}\end{array}$ \\
\hline \multicolumn{5}{c}{ FK Com } \\
Spot1 & \pm 1.61 & \pm 0.26 & \pm 0.12 \\
Spot2 & \pm 4.39 & \pm 5.55 & \pm 0.33 \\
Spot3 & \pm 5.71 & \pm 4.95 & \pm 0.60 \\
\multicolumn{5}{c}{ HD 199178 } \\
Spot1 & \pm 1.19 & \pm 0.42 & \pm 0.08 \\
Spot2 & \pm 3.07 & \pm 1.09 & \pm 0.38 \\
Spot3 & \pm 10.7 & \pm 5.85 & \pm 0.78 \\
\hline
\end{tabular}

functions $\sigma$ are plotted from Eq. (1) versus spot longitude (upper panel), spot latitude (middle), and spot size. These functions are approximated by polynomials so as to find the minimum, which corresponds to the best value of the respective parameter. In Table 7 , the uncertainties in spot longitude $\Delta l$, latitude $\Delta b$, and in size $\Delta r$ are shown, corresponding to a deterioration in the fitting by $\Delta \sigma=0.0001, \Delta \sigma$ being the triple standard error of the fitting polynomial. From Figs. 14, and 15, and Table 7, it is apparent that the large Spot 1 (for both stars) has well-determined position and size. For the smaller spots, on both FK Com and HD 199178, position and size are determined with larger uncertainties. Our accuracy analysis shows that the results discussed in the previous sections could be accepted with reasonable confidence.

\section{Conclusions}

For FK Com, we find conclusive evidence of a large highlatitude spot, whose longitude is oscillating between two positions, about $180^{\circ}$ apart. This spot is probably migrating: both longitudinal and latitudinal migration are possible. The migration of this spot (in either case) proceeds in an oscillatory manner with a cycle of $5.8 \pm 0.1$ years. The same cycle is also noticed in other characteristics of the light behaviour, i.e. in the variations in minimum light, mean light and possibly in the amplitude of the rotational modulation. This is a strong indication that the 5.8 years cycle is dominating the FK Com photometric behaviour. The solar cycle connection is a tempting possibility.
Occasionally, rapid flip-flops in activity are observed: three of them are probably caused by a sudden migration in longitude of the main Spot 1. Other flip-flops are caused by the emergence of another spot. However, after an occasional interruption by a flipflop, the oscillatory process continues. Light curve modelling reveals also two smaller spots at intermediate latitudes.

We have suggested a new "mean" photometric period for HD 199178, $P=3.30025$ and used it to study the photometric behaviour. The $\mathrm{O}-\mathrm{C}$ diagram and the variations in the longitude of Spot 1 show a cycle of 4.2 years. Both longitudinal and latitudinal migration of Spot 1 are possible and could account for the variations observed.

Acknowledgements. This work is partly supported by the UNESCO International Basic Sciences Program. We thank Prof. A. C. Cameron and Prof. A. Boksenberg for critically reviewing the manuscript. Thanks are due to both our referees for many fruitful remarks and suggestions!

\section{References}

Al-Naimiy, H. 1978, Ap\&SS, 53, 181

Berdyugina, H., \& Tuominen, I. 1998, A\&A, 336, L25

Berdyugina, S. V., Berdyugin, A. V., Ilyin, I., \& Tuominen, I. 1999, A\&A, 350, 626

Bopp, B. W. 1982, in Proc. Second Cambridge Workshop on Cool Stars, Stellar Systems, and the Sun, SAO Special Report, 392, 207

Bopp, B. W., \& Rucinski, S. M. 1981, in Fundamental Problems in the Theory of Stellar Evolution, ed. D. Sugimoto, D. G. Lamb, \& D. N. Schramm (Dordrecht: Reidel), IAU Symp., 93, 177

Bopp, B. W., \& Stencel, R. E. 1981, ApJ, 247, L131

Bopp, B. W., Africano, J. L., Stencel, R. E., Noah, P. V., \& Klimke, A. 1983, ApJ, 275, 691

Chugainov, P. F. 1966, IBVS, No. 172

Dorren, J. D., Guinan, E. F., \& McCook, G. P. 1984, PASP, 96, 250

Eker, Z. 1994, ApJ, 420, 373

Eker, Z. 1995, ApJ, 445, 526

Eker, Z. 1996, ApJ, 473, 388

Eker, Z. 1999a, ApJ, 512, 386

Eker, Z. 1999b, New Astr., 4, 365

Elstner, D., \& Korhonen, H. 2005, AN, 326, 278

Hackman1, T., Jetsu, L., \& Tuominen, I. 2001, A\&A, 374, 171

Herbig, G. H. 1958, ApJ, 128, 259

Heckert, P. A. 1993, IBVS, No. 3954

Huenemoerder, D. P. 1986, AJ, 92, 673

Huenemoerder, D. P., Ramsey, L. W., Buzasi, D. L., \& Nations, H. L. 1993, ApJ, 404, 316

Huovelin, J., Piirola, V., Vilhu, O., Efimov, Y. S., \& Shakhovskoy, N. M. 1987, A\&A, 176, 83

Jetsu, L., Huovelin, J., Tuominen, I., et al. 1990, A\&A, 236, 423

Jetsu, L., Pelt, J., Tuominen, I., \& Nations, H. 1991, The Sun and Cool Stars, Activity, Magnetism, Dynamos, ed. I. Tuominen, D. Moss, \& G. Rudiger, Springer, Proc. IAU Coll., 130, 381

Jetsu, L., Pelt, J., \& Tuominen, I. 1993, A\&A, 278, 449

Jetsu, L., Tuominen, I., Antov, A., et al. 1994a, A\&AS, 103, 183

Jetsu, L., Tuominen, I., Grankin, K. N., Mel'Nikov, S. Yu., \& Schevchenko, V. S. 1994b, A\&A, 282, L9

Jetsu, L., Tuominen, I., Bopp, B. W., et al. 1999a, A\&AS, 139, 513

Jetsu, L., Pelt, J., \& Tuominen, I. 1999b, A\&A, 351, 212

Korhonen, H., Berdyugina, S., Hackman, T., et al. 1999, A\&A, 346, 101

Korhonen, H., Berdyugina, S., Hackman, T., et al. 2000, A\&A, 360, 1067

Korhonen, H., Berdyugina, S., Tuominen, I., et al. 2001a, A\&A, 374, 1049

Korhonen, H., Berdyugina, S., Strassmeier, K., et al. 2001b, A\&A, 379, L30

Korhonen, H., Berdyugina, S., \& Tuominen, I. 2002, A\&A, 390, 179

Lenz, P., \& Breger, M. 2005, CoAst, 146, 53

Olivera, J. M., \& Foing, B. H. 1999, A\&A, 343, 213

Poe, C., \& Eaton, J. 1985, ApJ, 289, 644

Rucinski, S. 1991, AJ, 101, 2199

Strassmeier, K., Bartus, J., Cutispoto, G., \& Rodono, M. 1997, A\&AS, 125, 11

Strassmeier, K., Lupinek, S., Dempsey, R., \& Rice, J. 1999, A\&A, 347, 212

Walter, F. M., \& Basri, G. S. 1982, ApJ, 260, 735

Webbink, R. F. 1976, ApJ, 209, 289

Welty, A. D., \& Bopp, H. 1993, PASP, 105, 142

Welty, A. D., \& Ramsey, L. W. 1994, ApJ, 435, 848 